\title{
Un monde de papier, avec Erri De Luca et Silvia Acocella
}

\section{Monica Zanardo}

\section{(2) OpenEdition}

1 Journals

\section{Édition électronique}

URL : https://journals.openedition.org/genesis/4723

DOI : 10.4000/genesis.4723

ISSN : 2268-1590

\section{Éditeur :}

Presses universitaires de Paris Sorbonne (PUPS), Société internationale de génétique artistique littéraire et scientifique (SIGALES)

\section{Édition imprimée}

Date de publication : 15 décembre 2019

Pagination : $123-128$

ISBN : 979-10-231-0650-3

ISSN : 1167-5101

\section{Référence électronique}




\title{
Un monde de papier Entretien avec Erri De Luca et Silvia Acocella
}

\author{
Propos recueillis par Monica Zanardo
}

Erri De Luca (Naples, 1950) a publié de nombreux contes et romans, couronnés d'un franc succès. Dans un riche entretien accordé à Irène Fenoglio pour Genesis ( $\left.n^{\circ} 25,2005\right)$, Il avait décrit sa méthode de travail et son rapport à l'écriture. Depuis, de nombreux ouvrages ont vu le jour (citons : Au nom de la mère, Le Poids du papillon, Les poissons ne ferment pas les yeux, Le Tort du soldat, La Nature exposée...). Près de quinze ans après ce premier entretien, nous avons repris contact avec lui, et il a accepté de répondre par écrit à quelques questions sur sa conception de la notion d'auteur et sur son rapport à ses archives littéraires. Nous avons également sollicité Silvia Acocella, enseignante de littérature italienne à l'université de Naples «Federico II», qui s'occupe depuis 2011 des archives personnelles de De Luca, conservées à Rome avec l'Archivio Eduardo de Filippo et le fonds Lotta Continua, auprès de la Fondation Erri De Luca. Ce double entretien, où les archives littéraires d'un écrivain sont présentées par l'auteur lui-même et par son archiviste, nous offre un regard inédit sur l'atelier d'écriture de De Luca.

Monica Zanardo - Ceux qui ont lu vos entretiens savent très bien que vous refusez le titre d'auteur et parfois même celui d'écrivain : vous préférez vous définir, selon les cas, comme un «rédacteur» ou un "exécutant»1. Bref, vous préférez vous considérer comme un artisan plutôt que comme un artiste. Mais qu'est-ce qu'un auteur, alors, d'après vous?

Erri De Luca - Un auteur littéraire, c'est quelqu'un qui vend un texte et perçoit un droit d'auteur. Moi, je bénéficie de cet avantage, mais je préfère dire que c'est ma vie passée qui est l'auteure.

M. Z. - Votre rapport à l'écriture comme artisanat et comme écoute me fait penser au geste empreint de sacralité du moine qui peint une icône et ne s'en estime pas l'auteur ${ }^{2}$. Comment choisissez-vous les histoires à raconter?

E. D. L. - Je ne les choisis pas, dans le sens où je ne fais pas de liste pour les trier. Une histoire s'impose avec insistance dans mon oreille avec une voix et je commence à la transcrire au stylo sur un cahier. Je ne reconnais aucune sacralité dans cet exercice de divertissement.

M. Z. - Je souhaiterais insister sur l'importance que vous attribuez à la «voix». Vous avez déclaré que, lorsque vous rédigez un roman, la phase préparatoire principale consiste dans la modulation de votre ton de voix sur celui de l'histoire que vous décidez de raconter ${ }^{3}$. Faut-il y voir un désir de réactiver, à l'époque moderne, la dimension orale du conte?

E. D. L. - Dans mes histoires une voix raconte de l'intérieur, ce n'est pas une narration à la troisième personne, mais à la première. Ce sont des récits oraux, j'écoute et je transcris. Les virgules, les points, les retours à la ligne sont des indications de pauses. Je sais depuis mon enfance que la voix humaine est le plus puissant instrument de transmission des sentiments, les bons et les mauvais. J'arrivais à m'identifier physiquement à ce que disaient les histoires des adultes. C'est pourquoi mon écriture est orale et physique.

M. Z. - La dimension orale de votre écriture, la recherche d'une voix susceptible de passer par la page écrite, est-elle à

1. « $[\ldots]$ je ne me considère donc pas de la catégorie des inventeurs, donc des auteurs : je suis un rédacteur» (Erri De Luca, «Je ne suis pas un écrivain, je suis un rédacteur de variantes », entretien avec Irène Fenoglio, Genesis, $\mathrm{n}^{\circ} 25,2005$, p. 131-149: p. 133); «Je ne me considère pas comme un chef d'orchestre, je dirais plutôt un exécutant» (Io Donna, 23 novembre 2016, en ligne, nous traduisons).

2. La littérature est l' «art de raconter une histoire qui n'est pas nouvelle - aucune histoire ne l'est plus - avec la candeur d'un aveugle qui, revenant vers la lumière et transi d'émotion, l'aperçoit pour la première fois » (Alzaia, Milan, Feltrinelli, 1997, p. 92, nous traduisons).

3. «[...] je fais l'imitation de la voix, je suis la voix, je ne suis pas l'écriture, je suis dans la suite de la voix, je recherche le ton de voix » (entretien avec I. Fenoglio, art. cit., p. 136). 
mettre en relation avec votre père? Je pense à votre premier livre (1989), Non ora, non qui (Pas ici, pas maintenant), que votre père, devenu aveugle, n'était pas en mesure de lire, mais qu'il pouvait tout de même écouter. L'idée d'un destinataire qui ne peut qu' "écouter»votre écriture a-t-elle influencé dans une certaine mesure votre façon de rédiger vos histoires?

E. D. L. - Mon père écoutait la radio et ma mère lui lisait mes histoires. Avant qu'il meure, la radio a retransmis la lecture de cinq de mes courts récits, Les Coups des sens. À part le sens de la vue, il les percevait avec les quatre autres. Mon père m'a fait découvrir les livres dans mon enfance, je lui dois cet héritage.

M. Z. - Vos livres se prêtent bien à une lecture à voix haute, et vos pages, comme vous l'affirmez, "ne sont pas plus longues que le souffle dont on a besoin pour en prononcer les phrases ». S'agit-il simplement d'une métaphore, ou avezvous l'habitude de relire vos écrits à voix haute, pour en tester le rythme et pour en «écouter l'écriture»?

E. D. L. - Je marmonne pendant que j'écris, mais ensuite je ne relis pas, je recopie pour comprendre si la page me convient. Je revois ainsi mes lignes et je les réécoute.

M. Z. - Outre cette dimension orale du récit, dimension originelle vers laquelle votre écriture fait retour, dans une certaine mesure, vous accordez également une grande attention à la mise en page. Dans votre entretien avec Irène Fenoglio, vous avez déclaré que la forme définitive d'un texte consiste, pour vous, en une version tapuscrite, et que "dans cette dernière écriture, je vois la page. Il y a dans la page un dessin ${ }^{4}$ ». Vous arrive-t-il de modifier un texte après en avoir fait une version dactylographiée, voire d'y apporter de modifications substantielles à l'occasion des épreuves?

E. D. L. - Je corrige peu, je ne fais pas tourner en bourrique l'éditeur. Je tiens à ce que la page soit graphiquement aérée et non compacte avec des lignes serrées comme les rangs d'un défilé militaire. Il doit y avoir de l'air, la page doit avoir des pauses, permettre à celui qui lit de s'interrompre. Il m'arrive d'ajouter quelque chose quand je reçois les premières épreuves. Des mois se sont écoulés depuis la remise à l'éditeur et des idées que je n'avais pas eues pendant la rédaction me sont venues. J'ajoute donc quelque chose, mais je ne retire pas.

M. Z. - Vous évoquez souvent la riche bibliothèque de votre père et votre chambre d'enfant, où vous étiez entouré de livres : mais vous avez certainement enrichi, depuis, cette collection... Quel est le rapport entre ces deux bibliothèques (celle dont vous avez hérité et votre bibliothèque personnelle)? Estimez-vous qu'il existe une différence entre les livres qui vous ont, pour ainsi dire, trouvé (car ils étaient tout simplement dans votre chambre) et les livres que vous avez choisis, voire cherchés?

E. D. L. - Je suis plus lecteur qu'écrivain, j'ai lu plus de pages que je n'en ai écrites. En outre, je peux lire dans plusieurs langues, mais je n'écris qu'en italien. En lisant, j'ai eu des occasions de bonheur que je ne trouve pas en écrivant. Les livres de mon père, ce qui reste des déménagements, sont maintenant réunis avec les autres. Mais il achetait les siens, alors que les autres m'arrivent gratuitement. Les livres sont des rencontres, alors ils s'arrêtent, sinon ils me glissent des mains dès les premières pages. On ne peut pas réserver les rencontres, du moins celles que j'ai eues.

M. Z. - Dans Alzaia (1997), on peut lire : «Ce que je recherche dans les livres, c'est la lettre, ou même simplement la phrase qui a été écrite pour moi et que, pour cette raison, je souligne, je recopie, j'extrais et j'emporte ${ }^{5}$. Avez-vous l'habitude de souligner dans les livres que vous lisez? Cela vous arrive-t-il aussi de les annoter? Quand vous parlez $d$ ' extraction», doit-on supposer l'existence de cahiers d'extraits, destinés à héberger les phrases dont vous avez l'impression qu'elles ont été «écrites pour vous»?

E. D. L. - Je ne souligne pas, je recopie sur un cahier la phrase qui m'ouvre une nouvelle pensée. Je prends soin de noter le nom et le titre pour les citer au cas où je les utiliserais. Ces phrases n'ont pas un cahier à part, elles sont au milieu d'autres pages (fig. 1).

4. «Je ne suis pas un écrivain...», art. cit., p. 139.

5. E. De Luca, Alzaia, op. cit., p. 117. 


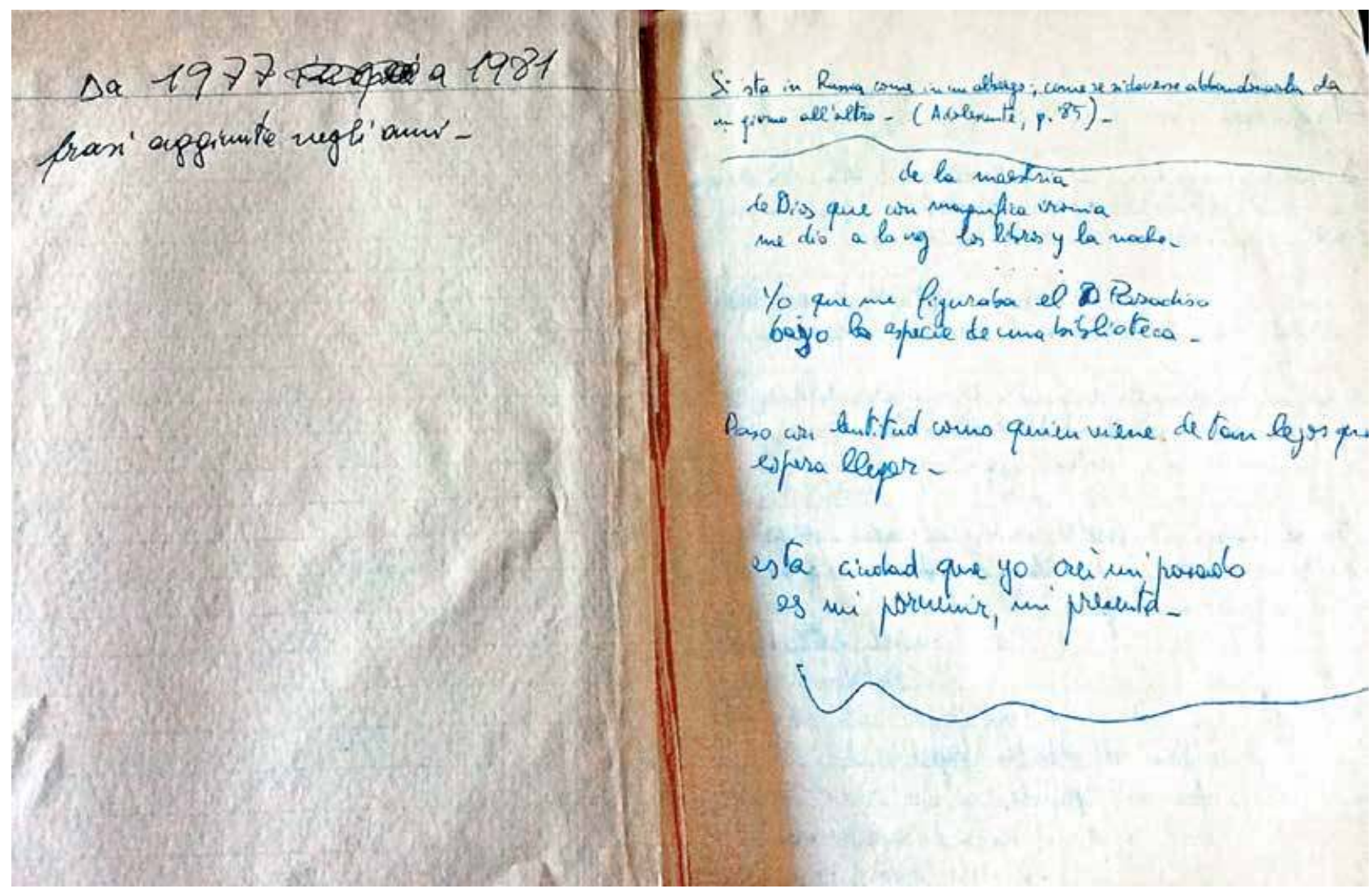

Fig. 1 : Erri De Luca, cahier manuscrit (1977-1981).

On peut lire sur cette page une citation tirée de L'Adolescent de Dostö̈evski (en traduction italienne)

et quelques vers du Poema de los dones de Borges.

(c) Rome, Fondazione Erri De Luca. Avec l'aimable autorisation de l'auteur

M. Z. - Silvia Acocella, quelles traces trouve-t-on dans les archives de la formation d'écrivain de De Luca et de son activité de lecteur?

Silvia Acocella - On trouve dans les archives quelques cahiers datant de l'école primaire (conservés par sa mère), des récits manuscrits de son père (conservés par Erri luimême), qui témoignent de l'attention portée par sa famille à la préservation de l'écrit, ou encore quelques journaux succincts, comportant de très brèves notations. Mais les premiers cahiers dignes d'intérêt pour la formation de son style sont ceux où Erri consigne des phrases tirées d'auteurs qu'il lisait, et qui ont marqué sa vie et son esprit. On lit ainsi, dans un cahier daté des années 1977-1981 où sont recueillies des citations accumulées au fil des ans, une phrase d'Hofmannsthal : «Il faut cacher la profondeur. Où? À la surface» (fig. 2). Elle eut une grande importance pour Erri, y compris pour ses «traductions de service» de la Mikrà6.
M. Z. - Dans un certain sens, ce recueil de pensées qu'est Alzaia pourrait être considéré comme un cahier d'extraits, d'où ressortent de nombreuses voix. Erri De Luca, comment est né ce livre?

E. D. L. - Au début, c'étaient des pages écrites pour le quotidien Avvenire qui les hébergeait au-dessus du titre du journal. Pendant quelques mois, j'en ai écrit une par jour. Puis, j'en ai choisi certaines et j'en ai ajouté d'autres. Le titre Alzaia est venu ensuite.

M. Z. - En plus de nombreux contes et romans, vous avez publié aussi plusieurs traductions. Y a-t-il des différences

6. La Bible, à laquelle De Luca préfère faire référence avec le mot juif «Mikrà » (c'est-à-dire «Lecture»). Par «traductions de service» l'on entend celles qui n'ont pas une visée éditoriale, et que l'auteur rédige pour son usage personnel. 
concrètes, dans votre méthode de travail, entre l'écriture et la traduction?

E. D. L. - En excluant le mot travail des écritures que je fais, les traductions viennent du sentiment d'admiration, tandis que l'écriture vient d'un enthousiasme.

M. Z. - Vos traductions connaissent-elles une phase de "décantation» analogue à celle qui caractérise, selon vos déclarations, l'écriture de vos romans ${ }^{7}$ ?

E. D. L. - Pour les traductions de l'hébreu ancien, je m'en tiens au rythme d'un seul vers par jour, en allant rechercher chaque mot dans tous les passages des Écritures où il se manifeste. Je cherche la biographie de chaque mot. Cette lenteur me suffit.

M. Z. - Silvia Acocella, trouve-t-on dans les archives des témoignages sur l'activité de De Luca traducteur? Si c'est le cas, quelles sont selon vous les spécificités de De Luca traducteur par rapport à De Luca narrateur?

S. A. - La traduction a accompagné pendant un certain nombre d'années l'écriture narrative, un mouvement de va-et-vient s'établissant entre l'une et l'autre. Les traductions ont renforcé certains choix terminologiques vers lesquels tendait naturellement le style d'Erri mais qui, par le jeu d'une confrontation avec les champs lexicaux de différentes langues, révélaient de nouvelles profondeurs.

\section{Z. - Comment est née l'idée de la Fondazione Erri De Luca, active depuis $2011^{8}$ ?}

E. D. L. - Du désir et de la volonté de sa présidente, Paola Porrini Bisson. Non pas tant pour conserver mes archives, mais pour s'occuper, à son niveau, de jeunes immigrés et de leurs études en italien.

\section{Z. - Silvia Acocella, pourriez-vous nous en dire plus ?}

S. A. - Lorsque la Fondazione Erri De Luca a été créée à Rome le 1er juillet 2011, j'ai été appelée par l'auteur en raison du lien profond qui nous unit : ce lien s'est tissé au cours d'expériences que nous avons partagées, depuis la guerre de Bosnie où j'étais assise à ses côtés dans le fourgon d'un convoi humanitaire, et s'est constamment enrichi de notre amour pour les livres. J'ai proposé de m'occuper de ses archives car j'ai eu la chance d'assister à la composition de quelques-unes des pages de ses romans et de feuilleter les cahiers qui s'accumulaient au fil des ans. J'étais consciente qu'il s'agissait là d'une documentation d'autant plus précieuse qu'elle attestait d'une pratique de plus en plus rare : qu'un écrivain rédige entièrement à la main la première version de ses romans. Il fallut du temps à Erri pour que le mouvement de sa plume sur le papier se cale sur le rythme de sa pensée; puis la plume s'est accordée comme un instrument, désormais irremplaçable. L'écrivain en parle lui-même sur une page de notre site à laquelle je vous renvoie :

Enfant, j'écrivais avec empressement, avec fureur. Je m'irritais contre la lenteur de ma main qui ne soutenait pas le flux des images et des pensées. Je tentais des abréviations sténographiques mais cela ne suffisait pas. Je relisais quelque temps après, puis je jetais. Par la suite, mes années de travail ouvrier m'ont inculqué du respect pour ces mains qui me rapportaient un salaire. Mon écriture a appris à faire de même, à suivre la cadence et le rythme de la main. Les phrases prenaient la longueur de la respiration. Je me rendis compte que je ne jetais pas. C'est ma main qui détermine à présent le flux du récit et qui le dirige. J'écris encore sur des cahiers à lignes, j'en conserve un grand nombre. C'est mon album, il me permet de savoir qui j'ai été, mieux que ne le feraient des photographies 9 .

M. Z. - Pouvez-vous nous donner quelques indications sur le contenu du fonds et sur son accessibilité?

S. A. - Le fonds Erri De Luca contient de nombreux cahiers, de petits agendas, des carnets et quelques cahiers grand format. Il n'est pas ouvert à la consultation tant que le travail d'archivage se poursuit. La numérisation des manuscrits

7. «Il faut que je le retrouve [le premier jet] avec une envie de le recopier. Si j'ai envie de recopier l'histoire à la main, alors c'est bon signe, cela signifie qu'elle me plaît encore», E. De Luca, «Je ne suis pas un écrivain...», art. cit., p. 140.

8. http://www.fondazionerrideluca.com/web/

9. Erri De Luca, «Mani», 3 octobre 2017 (http://fondazionerrideluca. com/web/mani/). 


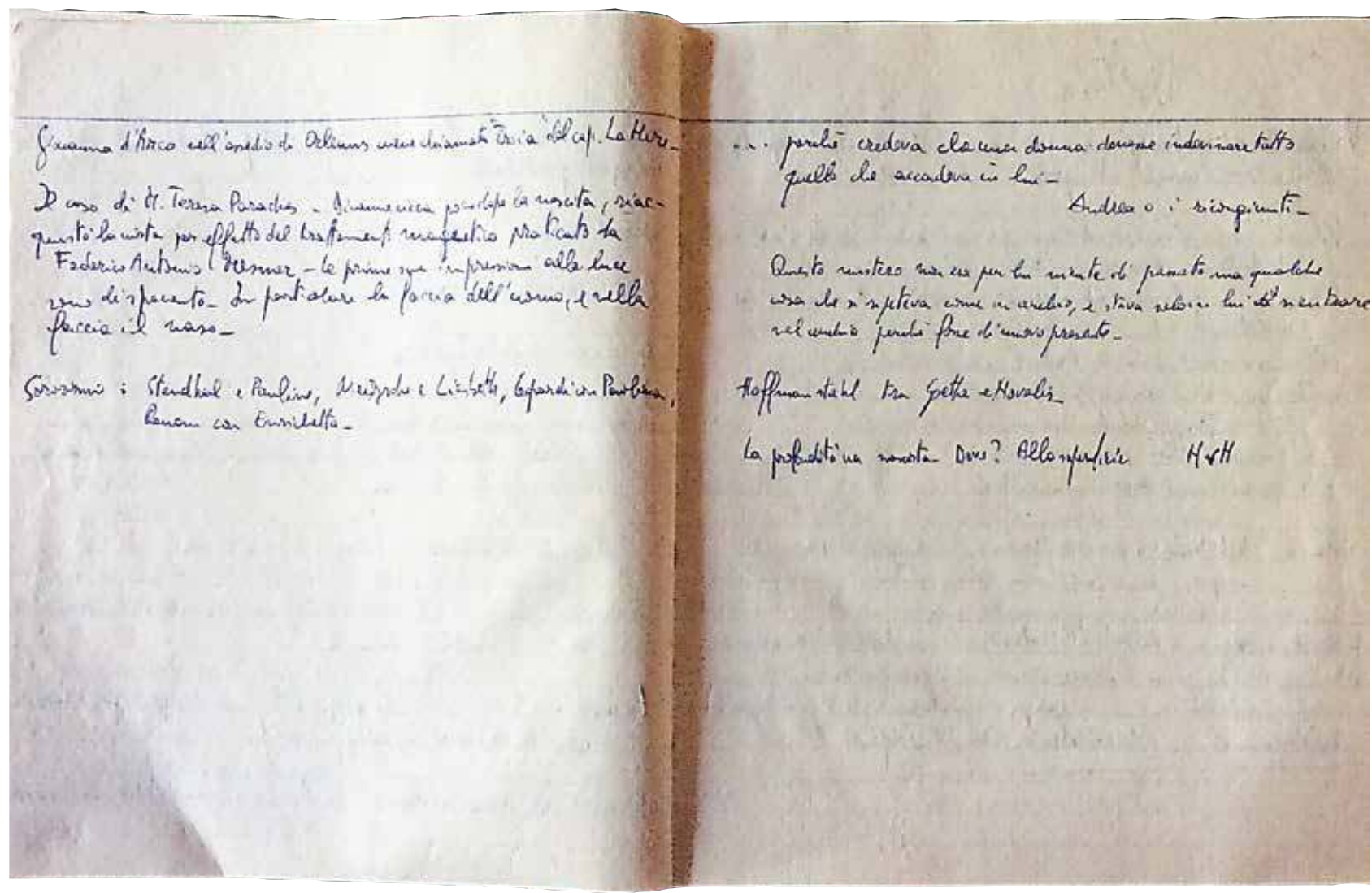

Fig. 2 : Erri De Luca, cahier de citations (1977-1981 env.)

() Rome, Fondazione Erri De Luca. Avec l'aimable autorisation de l'auteur

permettra un accès plus large et plus aisé aux chercheurs notamment étrangers.

M. Z. - Vous connaissez parfaitement ce précieux fonds manuscrit : quelles sont selon vous les principales pistes de recherche qu'offre une telle documentation?

S. A. - La requête de termes précis permise par la numérisation du fonds ouvrira de vastes possibilités de recherches car elle permettra d'étudier de façon diachronique l'espacement ou l'intensification des usages lexicaux propres à l'auteur et de prendre en considération ses travaux de traducteur. Il sera surtout possible, en considérant l'ensemble des cahiers réunis, d'observer la façon dont, lentement, les livres acquièrent leur format définitif, entre les lignes barrées et les segments entrelacés les uns aux autres. Erri envisage ses publications comme un «format réduit» des innombrables voix portées par le vent.

M. Z. - Erri De Luca, votre Fondation, parmi ses nombreuses activités, abrite aussi des archives. Y sont notamment conservés trois fonds : le fonds Lotta Continua, le fonds Eduardo De Filippo et le fonds De Luca. Entre mémoire et conservation, comment ces archives s'articulent-elles avec votre activité d'écriture?

E. D. L. - Nous avons mis en ligne la collection complète du quotidien Lotta Continua que mon père avait soigneusement conservée sous forme papier. Puis, j'ai reçu un don de matériel divers sur Eduardo De Filippo, consultable à la Fondation, dont le siège est chez moi. Un prix annuel a été créé pour récompenser une thèse de doctorat qui porte sur mon écriture. La Fondation, ce n'est pas moi, et au cours des deux années du procès intenté contre moi à Turin pour avoir soutenu la lutte du Val de Suse ${ }^{10}$, la Fondation s'est limitée à recueillir du matériel d'archives sur l'affaire.

10. Solidaire du mouvement NO-TAV, qui s'oppose à la construction de la ligne à grande vitesse Lyon-Turin, De Luca a été accusé en 2015 d' «incitation au sabotage» : son procès lui offrit l'occasion de défendre la liberté d'expression. Voir à ce sujet Anni di rame (2019). 
M. Z. - Silvia Acocella, comment décririez-vous le rapport de De Luca avec ses propres archives littéraires? L'auteur opère-t-il une sélection parmi ses documents (certains étant jugés privés, d'autres destinés à être librement consultés)?

S. A. - Erri tend par tempérament à ne pas accorder de prix à la documentation relative à son activité d'écriture; il tend par ailleurs à se considérer avant tout comme un lecteur plus que comme un écrivain. J'ai dû m'employer à le convaincre de réunir ses cahiers et de les mettre à l'abri, notamment de l'humidité. Cela dit, Erri m'a beaucoup aidée, dès le début, surtout pour leur datation. Un cahier important qui manifeste pour la première fois une intention d'auteur est daté de 1979 : il lui a été donné afin d'y réunir des récits jusqu'alors épars et inachevés. Avec le temps, l'écriture des cahiers a répondu à des stratégies plus précises, la page de gauche étant systématiquement laissée blanche pour les corrections. C'est ensuite qu'Erri a voulu faire cö̈ncider chaque cahier avec un seul roman : c'est le cas de Montedidio, qui est le fruit, plus qu'aucun autre de ses romans sans doute, d'une écriture de premier jet, sans plan préalable, mais d'un jet sorti au compte-gouttes de sa plume. Ma plus forte émotion, durant mon travail dans les archives, fut de reconnaître dans l'écriture désordonnée de différents cahiers la formation progressive de son tout premier roman, Acide, Arc-en-ciel, composé avant Pas ici, pas maintenant, mais publié trois ans seulement après celui-ci, en 1992. Assister à la naissance d'un écrivain, voir sa plume transcender, suivre le cours de ses pensées en composant la trame d'une histoire, voilà un spectacle naturel qui donne le sentiment, à quiconque a consacré sa vie à ce monde de papier, comme je l'ai fait, de se trouver soudain placé devant un vaste panorama.

M. Z. - Erri De Luca, comment décidez-vous des documents à garder et de ceux à détruire? Le choix est-il dicté par une sorte d' «intention d'archive», par un désir de laisser une trace (réfléchie et soigneusement étudiée) de votre activité d'écrivain?

E. D. L. - Je n'ai pas conservé les lignes qui ne me plaisaient pas. Mais comme j'écris sur des cahiers, j'en ai un beau paquet que pourront feuilleter ceux qui voudront jeter un coup d'œil à mon écriture...

Propos de De Luca traduits par Danièle Valin; propos de Silvia Acocella traduits par Pierre Musitelli 